\title{
Depression prevention program for workers of private companies: A randomized controlled trial
}

\author{
Mariko Kawaharada $^{1}$, Izumi Ueda ${ }^{2 *}$, Eiji Yoshioka ${ }^{3}$, Michiyo Hirano ${ }^{1}$, Tamiko Ikeno ${ }^{4}$, \\ Yukari Shindou ${ }^{1}$, Reiko Kishi ${ }^{4}$ \\ ${ }^{1}$ Department of Comprehensive Development Nursing, Faculty of Health Sciences, Hokkaido University, Sapporo, Japan \\ ${ }^{2}$ Graduate School of Health Sciences, Sapporo Medical University, Sapporo, Japan \\ ${ }^{3}$ Department of Health Science, Asahikawa Medical College, Asahikawa, Japan \\ ${ }^{4}$ Center of Environmental and Health Sciences, Hokkaido University, Sapporo, Japan \\ Email: *iueda@sapmed.ac.jo
}

Received 29 January 2013; revised 5 March 2013; accepted 14 March 2013

\begin{abstract}
Objective: The aim of this study was to examine the effects of a depression prevention program based on stress inoculation training (SIT) for workers of two private companies, by means of a randomized controlled trial. Method: Ninety three workers of two private companies were assigned to intervention and control groups, and two sessions were conducted in the intervention group of each company at an interval of four weeks. The sessions included lectures on responses to stress and coping skills, problem-solving training, cognitive restructuring training, relaxation, group discussions and self-monitoring. After completing the sessions, two follow-up letters were sent to the participants at two-month intervals. Data from 38 intervention subjects and 39 control subjects were analyzed. Results: There were no major differences between the variables of intervention subjects and control subjects. After two sessions, there was a marginally significant increase of seeking social support in the intervention group $(P=0.052)$ and a significant decrease of avoidance in both groups $(P<0.05)$. Concerning the depression score changes, the "Improvement" group of the intervention subjects showed an adjusted OR of 6.83 that was higher compared to the control group, indicating a marginally significant difference $(P=0.083)$. Conclusion: The results suggest the possibility that the SIT-based program of this study was effective for improving coping and preventing depression. Further research is necessary to develop a depression prevention program adjusted to gender, individual, occupational and other differences of subjects.
\end{abstract}

Keywords: Job Stress; Depression; Prevention; Stress

"Corresponding author.
Management; Intervention; Work-Place

\section{INTRODUCTION}

Depressive illness is projected to be the second leading cause of disability worldwide in 2020 [1]. Job stress is a risk factor for depression as well as for many other kinds of disorders [2,3]. The mental health condition of workers in Japan is deteriorating and mental health at workplaces is now becoming an important issue. In 2000, the Ministry of Health, Labor and Welfare established the "Guidelines for the promotion of worker's mental health in the workplace". The results of a survey carried out among Japanese companies show that the number of workers who suffer psychiatric disorders is increasing in about a half of the companies surveyed [4]. Therefore, mental health is an important workplace issue in Japan. Occupational health nurses conduct counseling for persons who experience problems with their mental health conditions, organize stress prevention education activities and perform other kinds of work to protect and promote mental health among workers at Japanese enterprises [5].

The development of stress research since the 1980s has given rise to numerous programs aimed at putting scientific achievements into practice, and intervention research on stress management for workers has also been carried out [6-8]. Murphy reviewed stress-management techniques used in workplace studies, and defined stressmanagement intervention as a technique designed to help employees modify their appraisal of stressful situations or deal more effectively with the symptoms of stress [6]. Murphy reported that training in cognitive-behavioral skills produced the most consistent effects on psychological outcomes, especially with regard to anxiety or anger. Van der Klink et al. conducted meta-analysis of randomized controlled trials designed to reduce job stress in workers with psychological problems, and reported 
that a cognitive-behavioral approach was the most effective in reducing complaints, improving the perceived quality of work and enhancing psychological resources and responses [7].

Stress management at the workplace is widely acclaimed, but the results of research into its effects on depression are insufficient [9]. A cognitive-behavioral therapy is known to be effective against psychiatric disorders including depression [10-12], but the effect of occupational stress management based on a cognitivebehavioral approach in preventing depression is unclear. Mino et al. carried out intervention among workers in high stress situations in the manufacturing industry, employing a combination of group sessions using cognitivebehavioral programs and individual counseling. As a result, depression improved in the intervention group, demonstrating that a stress management program at workplaces could help to reduce depression scores [9]. However, the development of stress management programs at workplaces is still rather limited. Along with improving working environments, it is necessary to provide health education offering ways of coping with stress and preventing development of depression among workers.

Stress inoculation training (SIT) is one of the major theories that support cognitive-behavioral therapy. It is a multidimensional intervention technique used both for treatment and prevention [13]. SIT consists of three overlapping stages: 1) conceptualization, 2) skill acquisition and rehearsal, and 3) application and follow-through. SIT aims at reducing and preventing stress response by increasing one's ability to cope with stress through the acquisition of coping skills and rehearsal of such skills in real life. The anxiety levels of teachers and nurses were lowered by SIT in previous studies conducted at workplaces $[14,15]$. In a study carried out among employees of insurance companies, SIT helped increase coping ability levels [16]. Those results suggest that SIT can be effectively used for depression prevention. In 2008, we carried out SIT-based stress management sessions among public servants and showed that SIT could improve stress coping skills [17]. However, that stress management intervention study had a number of limitations since it targeted only male workers at a public center, used no depression measurement nor was it a randomized controlled study. The aim of our present study was to examine the effects of a SIT-based depression prevention program conducted among male and female employees of two private companies through a randomized controlled trial.

\section{METHODS}

\subsection{Subjects and Study Design}

The subjects of this study were 51 (approximately 10\%) employees of a company involved in electrical work and 50 (approximately 10\%) employees of a telecommunications company. We asked several private companies especially those which employ occupational health nurse for their staff's well-being to participate this study. Two of them accepted. The employees of the companies received written explanations of the purpose and methods of the study and those who expressed their wish to participate were chosen as subjects. After excluding eight persons who were able to participate only for a limited period of time, the subjects of each company were randomly assigned to either an intervention group $\left(n^{1}=26\right.$, $\left.n^{2}=21\right)$ or control group $\left(n^{1}=25, n^{2}=21\right)$ using a table of random numbers.

Before starting the intervention in June, 2009, written explanations of the purpose and details of the research were distributed to the subjects along with questionnaires. Two sessions with the intervention subjects were carried with a four-week interval, and two follow-up letters were sent to the participants after completing the sessions at two-month intervals. Forty-four intervention subjects participated in the sessions, with 37 persons (84.1\%) attending both sessions. A post-test was conducted immediately after the sessions, and a follow-up test was conducted two months after the last letter was sent. Data from 38 intervention group members and data from 39 control group members who had returned all the questionnaires were analyzed (see Figure 1). In the following year, the same sessions were provided for the subjects in the control groups.

\subsection{Intervention Program}

The SIT-based depression prevention program of this study was developed by our research group. This program represented an improved version of our previous program and consisted of three phases: education, cognitive-behavioral skill development, and application [17]. To increase the participation rate, the number of sessions was decreased from three to two, and the length of each session was increased from $60 \mathrm{~min}$ to $90 \mathrm{~min}$. The sessions took place during office hours in the meeting rooms of the companies. They included lectures on responses to stress and coping skills, problem-solving training, cognitive restructuring training, relaxation, group discussions and self-monitoring diaries as homework between the sessions [18]. The occupational health nurses acted as instructors during the sessions. The instructors had acquired necessary skills through training organized by the research group. The instructors conducted the sessions based on the detailed health education guidance and textbooks developed by the research group. One member of the research group took part in the intervention as an assistant instructor. 


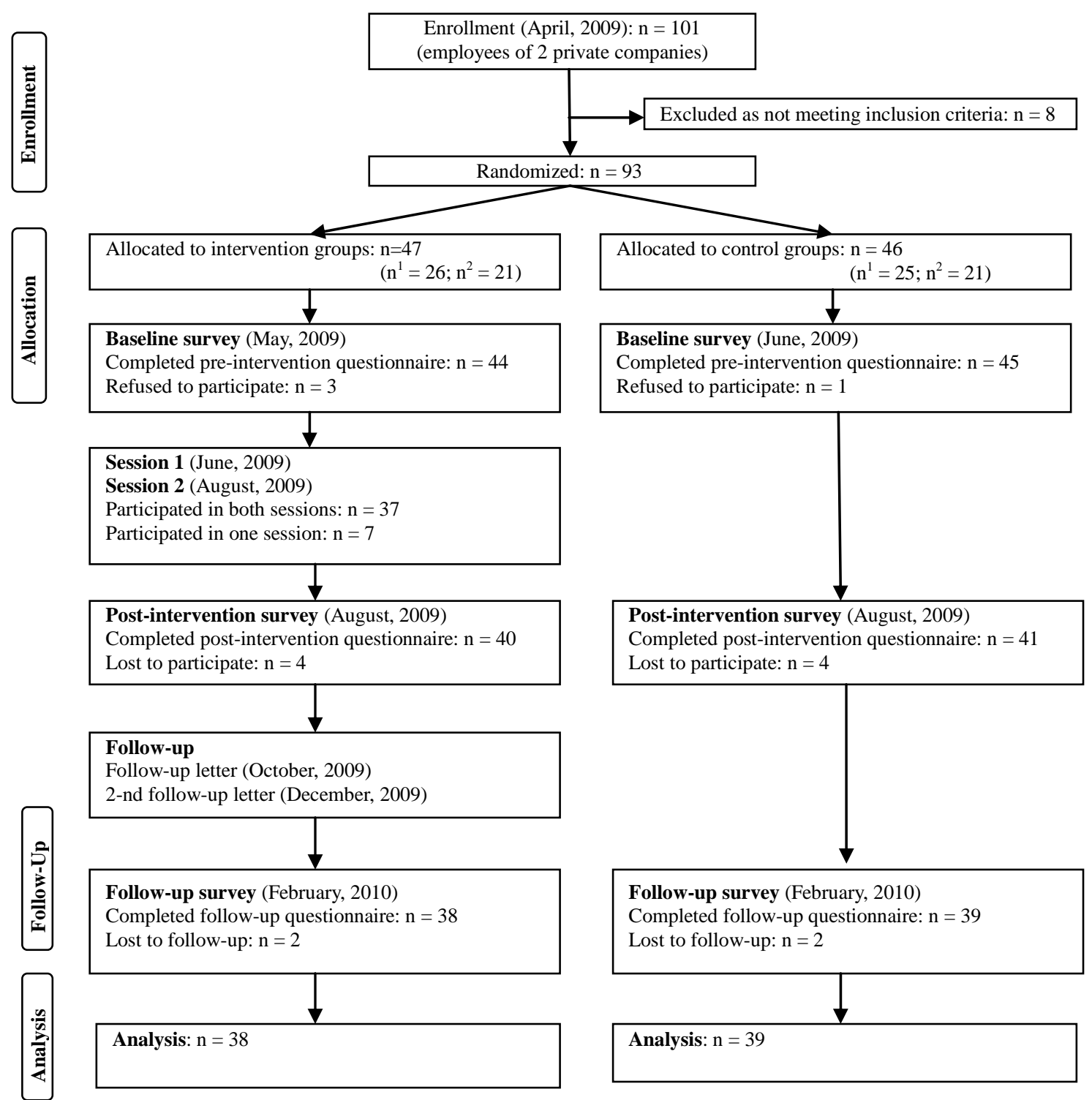

Figure 1. Flow of participants through trial.

\subsubsection{Session 1}

The purpose of the first session was to enhance the subjects' understanding of stress and help them acquire skills for coping with stress. The participants listened to a lecture on mental and physical reactions to stress and coping as an intermediate stress factor. Next, they learned skills that would help the participants cope with stress. It consisted of cognitive restructuring and training in problem-solving skills. During the cognitive restructuring, the participants learned to modify the thought that automatically occur to them in stressful situations and develop a well-balanced way of thinking using training sheets. Problem-solving training was conducted in pairs, and used the stressful experiences brought up the participants. The subjects were asked to record stressful experiences, their feelings, physical reactions, thoughts, coping ac- tions and the results of such actions, and whether any better alternatives could be used to cope with each situation.

\subsubsection{Session 2}

The second session was aimed at leaning to apply stresscoping skills, including relaxation and social support, in daily life. The participants listened to a lecture on the meaning and methods of relaxation and social support. Next, the subjects were divided into groups of four and engaged in group discussions on predicting stressful situations and effective methods of coping with them. The group discussion method helps develop more realistic and effective way of coping by enabling participants to learn from other people's coping experiences. Lastly, we advised the subjects to continue self-monitoring. 


\subsubsection{Follow-Up Letters}

After completing the sessions, two follow-up letters were sent at two-month intervals to encourage the participants to continue their stress management activities. The follow-up letters contained 15-item checklists on how the participants were using the methods of coping they had learned during the two sessions.

\subsection{Measurement Scales}

\subsubsection{Outcome Measures}

\section{1) Primary outcome measure}

Response to stress was assessed using 20 items from the Japanese version of the Center for Epidemiologic Studies for Depression Scale (CES-D) scored on a fourpoint Likert scale [19]. CES-D had been used as an outcome measure by Mino et al. to assess a stress-management program based on the cognitive behavioral approach and aimed to prevent depression among workers [9]. This study was based on SIT, one of the methods of cognitive behavioral approach, so we considered it appropriate to use CES-D for our stress-management program assessment. The score range is from 0 to 60 . A higher total score means a higher response to stress. Sixteen points or more indicate depression. Cronbach's alpha coefficient for CES-D was 0.86.

2) Secondary outcome measures

Coping was assessed using the Ways of Coping Checklist (WCCL) (47 items), scored on a four-point Likert scale (from 1 point to 4 points) [20]. The coping scale had the following six subscales: problem-solving (14 items), positive cognitive coping (10 items), seeking social support (6 items), wishful thinking (6 items), selfblame (4 items), and avoidance (7 items). Higher scores indicate a higher level of coping. Cronbach's alpha coefficient for WCCL was 0.88 .

\subsubsection{Other Measures}

Demographic information on the participants was obtained through questions on gender, age and marital status, regular exercise, working position and working hours per day. The questionnaire also contained items from the Japanese version of the sense of coherence scale (SOC) (13 items) to measure personal ability to manage psychological stressors [21].

\subsection{Statistical Analysis}

First, the baseline demographic and stress-related variables of the intervention and control subjects were analyzed using $\chi^{2}$ testing and t-testing. Second, intention to treat analysis was performed to ascertain the effects of intervention. These effects were evaluated by comparing depression and the stress-coping skills of the intervention and control subjects at the pre-, post- and follow-up sur- vey stages. One-way analysis of variance with repeated measurement and Bonferroni multiple comparison procedure were used to test the statistical significance of the intervention effects for each indicator.

Third, the changes in depression scores after the intervention were sorted into two groups: "Improvement" (depression (CES-D score 16 points and more)) changing to normal condition (CES-D score 15 points and less) and "No improvement". Then, logistic regression analysis was conducted to obtain odds ratios (OR) and 95\% confidence intervals (95\% CI) of each group before and after adjustment for workplace. The estimated prevalence OR of the "Improvement" group and its 95\% CI were calculated taking the control group as a reference group. A p value of $<0.05$ was considered to be significant. All analyses were conducted using the SPSS software Version 18 for Windows. A CONSORT checklist was used to improve the quality of the research $[22,23]$.

\subsection{Ethical Considerations}

To guarantee anonymity, each participant was asked to create a six-digit ID number and use it instead of his/her name when completing the questionnaires, which were submitted in sealed envelopes and opened directly by the researchers. The study was conducted with permission from and in line with the ethical regulations of the Board of Ethics for Medical Studies at Hokkaido University's Faculty of Health Sciences.

\section{RESULTS}

\subsection{Comparison of Pre-Survey Variables}

There were no significant differences in the demographic variables between the intervention and control subjects (Table 1). No significant difference was found in coping scores. The average depression scores showed a marginally significant difference, with 13.8 points $( \pm 6.7)$ for the intervention subjects and 11.0 points $( \pm 6.4)$ for the control subjects.

\subsection{Effects of Intervention among All Participants}

The pre- and post-intervention results for coping are presented in Table 2. As a result of the intervention, the scores for seeking social support in the intervention subjects showed a marginally significant increase after oneway analysis of variance with repeated measurement $(\mathrm{p}=$ 0.071 ) and a marginally significant increase after Bonferroni multiple comparison procedure for pre- and postintervention results $(p=0.052)$. The scores for avoidance showed a significant decrease after Bonferroni multiple comparison procedure for pre- and post-intervention results in both the intervention $(\mathrm{p}=0.044)$ and control $(\mathrm{p}=$ 
Table 1. Demographic variables of participants.

\begin{tabular}{|c|c|c|c|c|c|c|}
\hline & & \multicolumn{2}{|c|}{ Intervention group $(\mathrm{n}=38)$} & \multicolumn{2}{|c|}{ Control group (n = 39) } & \multirow{2}{*}{ p-value } \\
\hline & & $\mathrm{n}$ & $\%$ & $\mathrm{n}$ & $\%$ & \\
\hline Age (years) ${ }^{*}$ & & \multicolumn{2}{|c|}{$43.3( \pm 9.0)$} & \multicolumn{2}{|c|}{$42.2( \pm 9.8)$} & 0.609 \\
\hline \multirow{2}{*}{ Sex } & Male & 19 & 50.0 & 21 & 53.8 & 0.456 \\
\hline & Female & 19 & 50.0 & 18 & 46.2 & \\
\hline \multirow{2}{*}{ Marital status } & Married & 22 & 59.5 & 23 & 59.0 & 0.576 \\
\hline & Single or divorced & 15 & 40.5 & 16 & 41.0 & \\
\hline \multirow{2}{*}{ Occupation } & Clerk & 34 & 94.4 & 33 & 84.6 & 0.142 \\
\hline & Others & 2 & 5.6 & 6 & 15.4 & \\
\hline \multirow{3}{*}{ Position } & General office work & 22 & 57.9 & 22 & 56.4 & 0.987 \\
\hline & Middle management & 4 & 10.5 & 4 & 10.3 & \\
\hline & Higher management & 12 & 31.6 & 13 & 33.3 & \\
\hline \multicolumn{2}{|c|}{ Working hours per day* } & \multicolumn{2}{|c|}{$8.1( \pm 0.9)$} & \multicolumn{2}{|c|}{$8.1( \pm 0.9)$} & 0.603 \\
\hline \multirow{2}{*}{ Regular exercise } & Yes & 14 & 36.8 & 12 & 30.8 & 0.374 \\
\hline & No & 24 & 63.2 & 27 & 69.2 & \\
\hline \multicolumn{2}{|c|}{ Sence of coherence (SOC) } & \multicolumn{2}{|c|}{$54.5( \pm 9.4)$} & \multicolumn{2}{|c|}{$56.5( \pm 9.8)$} & 0.368 \\
\hline
\end{tabular}

${ }^{*}$ Means \pm SD. p values refer to the $\chi^{2}$ test for categorical variables or t-test for continuous variables.

0.011) subjects. There were no significant changes in depression scores between the intervention group and control group.

Table 3 shows intervention effects on CES-D for improvement change. In post survey, the adjusted OR of the "Improvement" group of the intervention subjects was higher compared to the control group, indicating a marginally significant difference.

\section{DISCUSSION}

After two sessions, the CES-D scores of the intervention group decreased, but the change was not significant. However, when the depression score changes were sorted into "Improvement" and "No improvement" groups, the "Improvement" group showed an adjusted OR of 6.83 that was higher compared to the control group, indicating a marginally significant difference. The result suggests a possibility of the intervention effect. Group education is less effective than individual coaching, but on the other hand it targets a large number of people and thus plays an important role as preventive education. Though the depression prevention program of this study only included two sessions there is a possibility it had a certain positive influence on many participants.

Individual differences in stress response depend largely on differences in coping $[13,24]$. According to the Job Demands-Control-Social support model, social support by seniors and coworkers is an important stress factor, apart from job demand and job control. Social support in the workplace can diminish the influence of stressors [25]. The number of intervention subjects seeking social support increased in the result of the intervention. One of the reasons explaining the increase might be the effect of the lecture about the importance of social support for coping with stress. In cases where changing a stressful situation is difficult, such as the death of a loved one, temporary avoidance of the stressor is said to be an effective method. However, long-term avoidance could possibly drag out the stressful situation and damage health $[13,20]$. In a survey conducted by Takaya et al. among workers in Japanese private companies, factor analysis was used to categorize coping attributes into problem-focused coping, emotion-focused active coping and escape-avoidance coping and it was shown that less avoidance led to less stress response [26]. In this study, avoidance scores decreased significantly after two sessions both in the intervention and control subjects. These results suggest that the intervention might have helped to reduce avoidance in subjects. The control group did not participate in the sessions, but they, probably, got influenced by completing the self-check questionnaires on depression and coping scales and knowing that mental health sessions were held in their workplace.

In our previous study using a SIT-based program, we reported a significant increase of problem-solving and acknowledgement in the result of the intervention among male office workers in the civil service [17]. This study targeted female and male workers in the private sector and demonstrated no changes in the above-mentioned 
Table 2. Intervention effects on stress coping.

\begin{tabular}{|c|c|c|c|c|c|}
\hline \multirow{2}{*}{ Variables } & & \multicolumn{2}{|c|}{ Intervention group $(\mathrm{n}=38)$} & \multicolumn{2}{|c|}{ Control group $(\mathrm{n}=39)$} \\
\hline & & Mean (SD) & $p$-value & Mean (SD) & $p$-value \\
\hline \multicolumn{6}{|c|}{ Depression (CES-D) } \\
\hline & Pre & $13.8( \pm 6.7)$ & 0.544 & $11.0( \pm 6.4)$ & 0.487 \\
\hline & post & $12.6( \pm 8.5)$ & & $11.7( \pm 6.0)$ & \\
\hline & follow-up & $13.9( \pm 8.6)$ & & $11.9( \pm 5.9)$ & \\
\hline \multicolumn{6}{|c|}{ Problem-solving } \\
\hline & Pre & $40.0( \pm 6.6)$ & 0.278 & $38.0( \pm 6.3)$ & 0.740 \\
\hline & post & $40.5( \pm 5.0)$ & & $38.7( \pm 6.9)$ & \\
\hline & follow-up & $40.8( \pm 6.2)$ & & $38.4( \pm 7.2)$ & \\
\hline \multicolumn{6}{|c|}{ Positive cognitive coping } \\
\hline & Pre & $28.5( \pm 4.4)$ & 0.462 & $28.2( \pm 3.6)$ & 0.367 \\
\hline & post & $29.1( \pm 4.7)$ & & $27.8( \pm 4.0)$ & \\
\hline & follow-up & $29.3( \pm 4.1)$ & & $28.5( \pm 3.3)$ & \\
\hline \multicolumn{6}{|c|}{ Seeking social support } \\
\hline & Pre & $16.7( \pm 4.0)^{\dagger}$ & 0.071 & $16.8( \pm 4.1)$ & 0.535 \\
\hline & post & $18.0( \pm 4.4)^{\dagger}$ & & $16.7( \pm 4.4)$ & \\
\hline & follow-up & $17.2( \pm 4.5)$ & & $17.2( \pm 4.8)$ & \\
\hline \multicolumn{6}{|l|}{ Self-blame } \\
\hline & Pre & $9.3( \pm 3.2)$ & 0.398 & $9.8( \pm 234)$ & 0.467 \\
\hline & post & $9.2( \pm 2.7)$ & & $9.3( \pm 2.7)$ & \\
\hline & follow-up & $8.7( \pm 2.6)$ & & $9.7( \pm 2.7)$ & \\
\hline \multicolumn{6}{|c|}{ Wishful thinking } \\
\hline & Pre & $11.9( \pm 3.8)$ & 0.421 & $12.4( \pm 3.4)$ & 0.171 \\
\hline & post & $11.2( \pm 3.6)$ & & $12.8( \pm 3.9)$ & \\
\hline & follow-up & $11.5( \pm 3.2)$ & & $11.9( \pm 3.7)$ & \\
\hline \multicolumn{6}{|l|}{ Avoidance } \\
\hline & Pre & $14.7( \pm 3.0)^{*}$ & 0.105 & $14.5( \pm 3.1)^{*}$ & 0.066 \\
\hline & post & $13.8( \pm 3.5)^{*}$ & & $13.7( \pm 3.5)^{*}$ & \\
\hline & follow-up & $14.1( \pm 3.4)$ & & $13.3( \pm 3.1)$ & \\
\hline
\end{tabular}

Means \pm SD. $p$ values refer to one-way analysis of variance. ${ }^{\dagger} \mathrm{p}<0.1,{ }^{*} \mathrm{p}<0.05$.

Table 3. Intervention effects on CES-D for improvement change.

\begin{tabular}{|c|c|c|c|c|c|c|c|c|}
\hline \multirow{2}{*}{ Post survey } & \multirow[t]{2}{*}{$\mathrm{n}$} & \multirow[t]{2}{*}{ Improvement } & \multicolumn{2}{|c|}{ Unadjusted OR (95\%CI) } & \multirow[t]{2}{*}{ p-value } & \multicolumn{2}{|c|}{ Adjusted OR (95\%CI) } & \multirow[t]{2}{*}{ p-value } \\
\hline & & & & & & & & \\
\hline Control group & 20 & 1 & 1.00 & & & 1.00 & & \\
\hline Intervention group & 26 & 6 & 6.94 & $(0.79-60.71)$ & 0.080 & 6.83 & $(0.78-59.93)$ & 0.083 \\
\hline \multicolumn{9}{|l|}{ Follow-up survey } \\
\hline Control group & 17 & 1 & 1.00 & & & 1.00 & & \\
\hline Intervention group & 21 & 4 & 4.75 & $(0.51-44.67)$ & 0.173 & 4.88 & $(0.52-46.22)$ & 0.167 \\
\hline
\end{tabular}

Adjusted by workplace. 
ways of coping. The differences in the results of the two studies may be explained by the differences in the subjects and session structures. Umanodan et al. conducted a multi-component worksite stress management training (SIT) among workers of a Japanese steel company that included six 30-minute sessions, with one session per month [27]. In per-protocol analyses of those who attended all sessions, significant favorable effects were observed on psychological distress, suggesting that stress prevention education is effective for preventing stress even with short sessions if they are carried out continuously. Our previous study included three sessions, but only half of the subjects participated in all of them [17]. In this study we held two sessions and the participation rate increased to $84.1 \%$. In addition, at the third SIT stage of application and follow-through the participants received two follow-up letters concerning stress management [13]. This study did not demonstrate any evident effect on depression prevention but it definitely stimulated the participants' stress management activities.

The limitations of this study must also be mentioned here. First, both the intervention and control subjects were chosen from the same sections, which might have made it possible to exchanged information that influenced the results in the control groups. In our further research we need to take measures such as randomized sampling using sections, departments or branches as blocks etc. to prevent the influence of intervention on control subjects. Secondly, the sample size was small. We had planned to recruit subjects from three companies but only two companies agreed to cooperate. Besides, the sample from each company also was rather small and the data of the male and female participants from both companies were put together in order to perform analysis by gender. Thirdly, while no differences were found in the variables between the intervention and control groups of the same company, the differences between the companies were not examined. The existence of such differences could have affected the results. Fourthly, the validity of the measurement was insufficient. Depression is only one of the numerous reactions triggered by stress. A measurement dealing with multiple aspects of stress is needed to thoroughly assess effects of an intervention [6].

\section{CONCLUSIONS}

The SIT-based depression prevention program within this study resulted in a marginally significant increase of seeking social support in the intervention participants and a significant decrease of avoidance after two sessions in the intervention and control participants. There was no significant difference between the depression scores before and after the intervention, indicating no intervention effect on depression. However, when the depression score changes were divided into "Improvement" and "No improvement" groups, the "Improvement” group showed an adjusted OR of 6.83 after two sessions that was higher compared to the control group, indicating a marginally significant difference. The results suggest the possibility that the SIT-based program of this study was effective for improving coping and preventing depression.

Further research in this field is necessary for developing a simple and effective educational program adjusted to individual, occupational and other differences to prevent depression and other mental health problems among Japanese workers.

\section{ACKNOWLEDGEMENTS}

We would like to thank the occupational health nurses and employees of the targeted companies for their participation in this study. This study was supported by the National Science Foundation of Japan.

\section{REFERENCES}

[1] Murray, C.J. and Lopez, A.D. (1997) Global mortality, disability, and the contribution of risk factors: Global burden of disease study. Lancet, 349, 1436-1442. doi:10.1016/S0140-6736(96)07495-8

[2] Stansfeld, S.A., Fuhrer, R., Shipley, M.J. and Marmot, M.G. (1999) Work characteristics predict psychiatric disorder: Prospective results from the Whitehall 2 study. Occupational Environment Medicine, 56, 302-307. doi:10.1136/oem.56.5.302

[3] Xu, W., Hang, J., Cao, T., Shi, R., Zeng, W., Deng, Y., Gao, W., Zhao, Y. and Guo, L. (2010) Job stress and carotid intima-media thickness in Chinese workers. Journal of Occupational Health, 52, 257-262. doi:10.1539/joh.L9157

[4] Mental Health Research Institute (2010) FY2010 White paper on mental health of industrial workers. Japan Productivity Center for Socio-Economic Development, Tokyo (in Japanese).

[5] Japanese Ministry of Health, Labor and Welfare (2000) Guideline for promoting mental health of workers at enterprises. Japanese Ministry of Health, Labor and Welfare, Tokyo (in Japanese).

[6] Murphy, L.R. (1996) Stress management in work settings: A critical review of the health effects. American Journal of Health Promotion, 11, 112-135. doi:10.4278/0890-1171-11.2.112

[7] van der Klink, J.J.L., Blonk, R.W., Schene, A.H. and van Dijk, F.J.H. (2001) The benefits of interventions for workrelated stress. American Journal of Public Health, 91, 270276. doi:10.2105/AJPH.91.2.270

[8] Deborah, L.J., Tanigawa, T. and Stephen, M.W. (2003) Stress management and workplace disability in the US, Europe and Japan. Journal of Occupational Health, 45, 1-7. doi:10.1539/joh.45.1 
[9] Mino, Y., Babazono, A., Tsuda, T. and Yasuda, N. (2006) Can stress management at the workplace prevent depression? A randomized controlled trial. Psychotherapy and Psychosomatics, 75, 177-182. doi:10.1159/000091775

[10] Beck, A.T. (1993) Cognitive therapy: Past, present, and future. Journal of Consulting and Clinical Psychology, 61, 194-198. doi:10.1037/0022-006X.61.2.194

[11] Butler, A.C., Chapman, J.E., Forman, E.M. and Beck, A.T. (2006) The empirical status of cognitive-behavioral therapy: A review of meta-analyses. Clinical Psychology Review, 26, 17-31. doi:10.1016/j.cpr.2005.07.003

[12] Paykel, E.S., Scott, J., Cornwall, P.L., Abbott, R., Crane, C., Pope, M. and Johnson, L. (2005) Duration of relapse prevention after cognitive therapy in residual depression: Follow-up of controlled trial. Psychological Medicine, 35, 59-68. doi:10.1017/S003329170400282X

[13] Meichenbaum, D.H. (1989) Stress Inoculation Training. Plenum Press, New York.

[14] Sharp, J.J. and Forman, S.G. (1985) A comparison of two approaches to anxiety management for teachers. Behavioral Therapy, 16, 370-383. doi:10.1016/S0005-7894(85)80004-6

[15] West, D.L., Horan, J.J. and Games, P.A. (1984) Component analysis of occupational stress inoculation applied to registered nurses in an acute care hospital setting. Journal of Consulting and Clinical Psychology, 31, 209-218.

[16] Gronningsaeter, H., Hytten, K., Skauli, G., Christensen, C.C. and Ursin, H. (1992) Improved health and coping by physical exercise or cognitive behavioral stress management-training in a work-environment. Psychology and Health, 7, 147-163. doi:10.1080/08870449208520016

[17] Kawaharada, M., Yoshioka, E., Saijo, Y., Fukui, T., Ueno, T. and Kishi, R. (2009) The effects of a stress inoculation training program for civil servants in Japan: A pilot study of a non-randomized controlled trial. Industrial Health, 47, 173-182. doi:10.2486/indhealth.47.173

[18] Greenberger, D. and Padesky, C.A. (1995) Mind over mood: Change how you feel by changing the way you think. Guilford Press, New York.

[19] Shima, S., Shikako, T., Kitamura, T. and Asai, M. (1985) New self-rating scales for depression. Psychiatry, 27, 717-723 (in Japanese).

[20] Nakano, K. (1991) The role of coping strategies on psychological and physical well-being. Japanese Psychological Research, 33, 160-167.

[21] Antonovsky, A. (1987) Unraveling the mystery of health: How people manage stress and stay well. Jossey-Bass Publishers, San Francisco.

[22] Tango, I. (2003) Randomized controlled trial: Design and statistical analysis. Asakurashoten, Tokyo (in Japanese).

[23] Schulz, K.F., Altman, D.G. and Moher, D. (2010) CONSORT 2010 statement update guideline for reporting parallel group randomized trials. British Medical Journal, 340, c332. doi:10.1136/bmj.c332

[24] Folkman, S. and Lasarus, R. (1985) If it changes it must be a process: Study of an emotion and coping during three stage of a college examination. Journal of Personality and Social Psychology, 48, 150-170. doi:10.1037/0022-3514.48.1.150

[25] Jonson, J.V. and Hall, E.M. (1988) Job strain, work place social support, and cardiovascular disease: A cross-sectional study of a random sample of the Swedish working population. American Journal of Public Health, 78, 13361342. doi:10.2105/AJPH.78.10.1336

[26] Takaya, M. and Hasegawa, Y. (2010) An analysis of the relation between stress coping profile and job stress using structural equation modeling (SEM). Sangyo Eiseigaku Zasshi, 52, 209-215 (in Japanese). doi:10.1539/sangyoeisei.E9011

[27] Umanodan, R., Kobayashi, Y., Nakamura, M., Kitaoka, K., Kawakami, N. and Shimazu, A. (2009) Effects of a worksite stress management training program with six short-hour sessions: A controlled trial among Japanese employees. Journal of Occupational Health, 51, 294-304. doi:10.1539/joh.L8055 